\title{
Processing Induced Change of Thermal Transport Properties of NiTi-Shape Memory Alloy
}

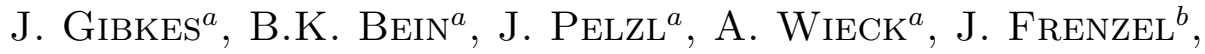 \\ G. EgGeler ${ }^{b}$, I. Delgadillo-Holtfort ${ }^{c}$ And M. Chirtoc ${ }^{d}$ \\ ${ }^{a}$ Institut für Experimentalphysik, Ruhr-Universität, D-44801 Bochum, Germany \\ ${ }^{b}$ Institute for Materials Science and Engineering, Ruhr-University \\ D-44801 Bochum, Germany \\ ${ }^{c}$ Instituto de Física de la Universidad de Guanajuato, IFUG \\ Apdo. E-143 León, Gto., Mexico \\ ${ }^{d}$ LTP, UTAP, Université de Reims, BP 1039, 51687 Reims Cedex 2, France \\ Photothermal radiometry was applied to investigate the influence of a \\ rolling process on the thermal properties of NiTi foils. The thermal diffusiv- \\ ity and thermal effusivity of the foils were determined at room temperature \\ from the frequency variation of the photothermal signals in thermal trans- \\ mission. Measurements were conducted on unrolled, rolled and thermally \\ annealed samples. The thermal diffusivity and thermal effusivity are found \\ to decrease considerably on rolling which is attributed to the influence of the \\ rolling induced lattice defects.
}

PACS numbers: $62.20 . \mathrm{Fe}, 78.20 . \mathrm{Nv}, 83.50 .-\mathrm{v}$

\section{Introduction}

For a large variety of applications of the shape memory alloy NiTi thermomechanical processed materials such as foils are used as the starting material [1]. Foils are in general produced from bulk alloy samples by rolling which is a deformation process where the force or stress is applied via the radial pressure of rolls. This deforms the metal and pulls it through the roll gap. When a metal is cold worked, microscopic defects are nucleated throughout the deformed area. These defects can be either point defects (a vacancy on the crystal lattice) or a line defect (an extra half plane of atoms jammed in a crystal). From the metalurgical point of view rolling results in a hardening of the metal and in a large decrease in ductility as defects accumulate through deformation and the slip or the move- 
ment of defects less frequently take place [2]. In the case of the shape memory alloys, in addition, the shape memory properties are altered. The phase transition temperatures between martensite and austenite state and the correlated hysteresis loop are changed or suppressed. The elastic and the thermal transport properties which are important for the functioning of the shape memory (SM) device will be different for different rolling processes and time. In this work we have studied the impact of cold rolling of foils of NiTi shape memory alloys on the thermal transport properties, the effusivity and the diffusivity of the treated foil.

\section{Experimental}

The samples under investigation were sheets of polycrystalline NiTi alloys with nearly equi-atomic composition. The sheets were produced with a commercial roller mill at room temperature. The thickness of the foils after rolling was measured mechanically. The thickness of the unrolled foil was about $1.67 \mathrm{~mm}$. The foil that had passed the final rolling treatment was measured after rolling and also after an annealing treatment at $800^{\circ} \mathrm{C}$ for about 15 min.

The thermal transport properties were studied with frequency dependent photothermal infrared radiometry (PTR). For the excitation of the thermal waves in the sample, the beam of an argon ion laser with a beam power of up to $600 \mathrm{~mW}$ and a diameter of about $2 \mathrm{~mm}$ was intensity-modulated with the help of an acoustooptical modulator in the frequency interval between $0.1 \mathrm{~Hz}$ and $100 \mathrm{kHz}$. The PTR experiments were performed in reflection as well as in transmission configuration and were conducted at room temperature. The signals were normalized (index $n$ ) with those measured from a glassy carbon (sigradur) flat disc.

\section{Results and discussion}

\subsection{Frequency dependent signal phases and amplitudes}

The normalized PTR signals measured in transmission were described analytically in the frame of a single layer model providing a mean diffusivity value. The signals measured in reflection were analyzed on the basis of a multilayer model. Here we report only on the results obtained in the transmission configuration which were analyzed in a single layer model because of the poor signal to noise ratio. Figure 1 shows the frequency variation of the phase and amplitude in transmission for an unrolled, a rolled and a thermally treated rolled sample. Above about $30 \mathrm{~Hz}$ the noise dominates the signal therefore only the low frequency range has been taken into consideration for the theoretical analysis. Adjustment to the phase data yield the thermal diffusivity values and the adjustment to amplitude data the thermal effusivity values for different rolling impacts. The obtained thermal parameters are displayed in Fig. 2, where the diffusivity values and the relative effusivity values are plotted versus the relative thickness change of the foil. The effusivity values are not corrected for different optical parameters of the sample and of the normalizer. 

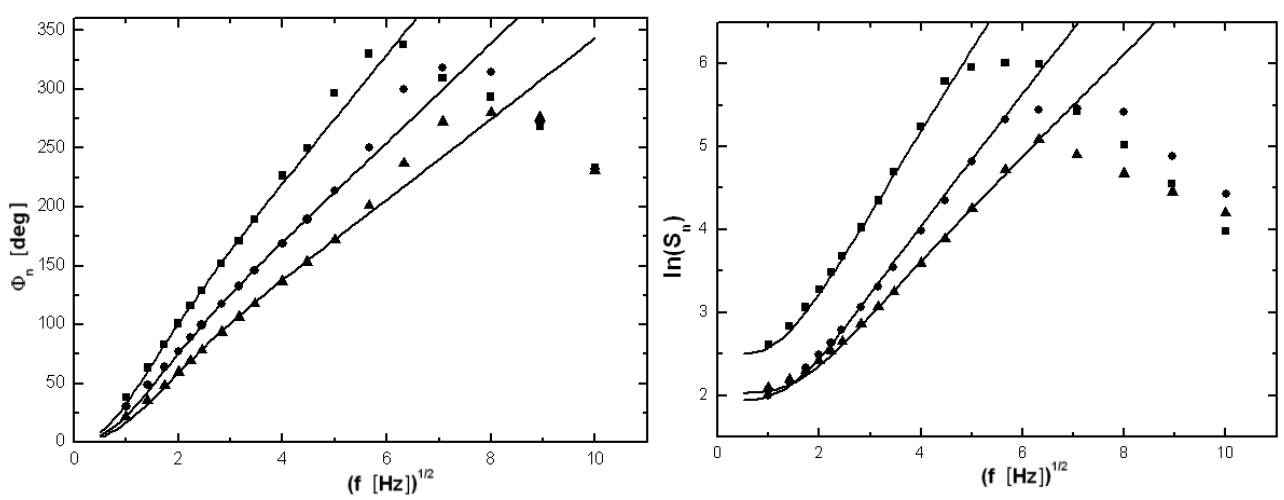

Fig. 1. Variation of the normalized absolute phase shift (left) and of the amplitude (right) as a function of the square root of the modulation frequency for an unrolled sample (squares), for a rolled sample (circles) and of the heat treated rolled sample (triangles). The theoretical curves represent the adjustment of a one-dimensional heat transport in a single layer model. The fit parameters are discussed below.

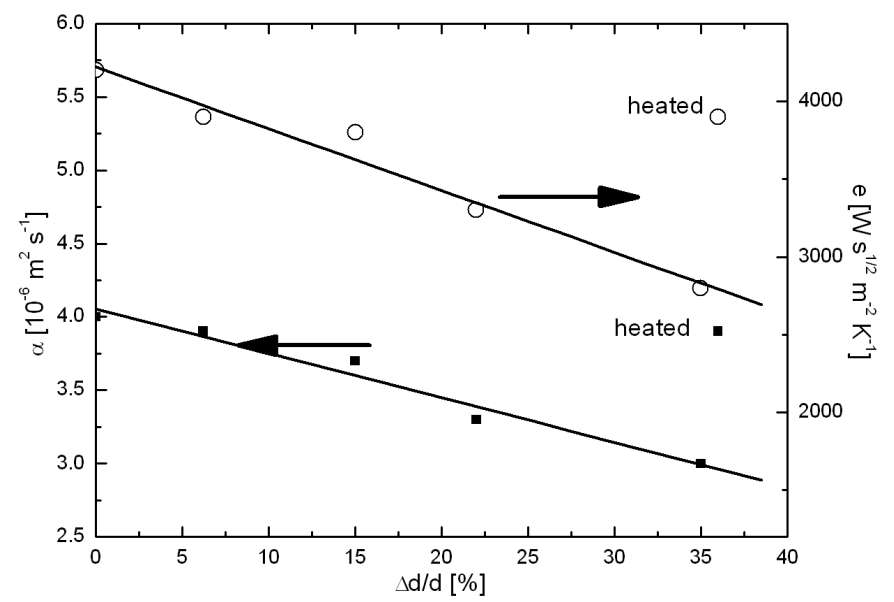

Fig. 2. Variation of the therm diffusivity (squares) and of the thermal effusivity (circles) as a function of the relative thickness change $d / d$ in $\%$ of the NiTi foil due to rolling. The data point marked with heated is the value obtained after heat treatment of the rolled sample.

\subsection{Discussion}

Both, the diffusivity $\alpha$ and the effusivity $e$ decrease with the rolling impact. Thermal annealing recovers fully the thermal parameters at the smaller thickness of the foil. Within the experimental uncertainty the decrease in $\alpha$ and $e$ are linear with $\mathrm{d} / \mathrm{d}: \alpha\left[10^{-6} \mathrm{~m}^{2} \mathrm{~s}^{-1}\right]=4.02-2.93(\mathrm{~d} / \mathrm{d})$ and $e\left[\mathrm{~W} \mathrm{~s} \mathrm{~s}^{1 / 2} \mathrm{~m}^{-2} \mathrm{~K}^{-1}=\right.$ $4175-3875(\mathrm{~d} / \mathrm{d})$. With the relations: $\alpha=\kappa / \rho c$ and $e=(\kappa \rho c)^{1 / 2}$ the variation of the thermal conductivity $\kappa$ and the mass specific thermal capacity $\rho c$ as a 
function of the rolling thickness can be determined. On rolling from $\mathrm{d} / \mathrm{d}=0$ to $\mathrm{d} / \mathrm{d}=0.35$ the thermal conductivity is reduced from $8.4 \mathrm{~J} /(\mathrm{kg} \mathrm{K})$ to $4.9 \mathrm{~J} /(\mathrm{kg} \mathrm{K})$ whereas simultaneously the mass specific thermal capacity $\rho c$ is changing from $2.05 \mathrm{~J} /\left(\mathrm{m}^{3} \mathrm{~K}\right)$ to $1.6 \mathrm{~J} /\left(\mathrm{m}^{3} \mathrm{~K}\right)$.

The reduction of the thermal conductivity by about $40 \%$ has to be attributed to the rolling induced lattice defects. In particular, dislocations that are produced and which are mostly oriented in plane act as internal interfaces with a thermal interface resistance for the heat transport perpendicular to the foil plane. The absolute value of the thermal conductivity of the unrolled sample fits into the lower end of the range of published values [1]. Thermal diffusivities as small as that of the rolled samples have been observed in NiTi films [3] pointing towards an important influence of the lattice defects also in these type of materials. The $\rho c$ value for the unrolled sample $\left(\rho c=2.05 \mathrm{~J} /\left(\mathrm{m}^{3} \mathrm{~K}\right)\right)$, however, is considerable lower than the value determined from the independently measured mass density and specific heat capacity $\left(\rho c=3.5 \mathrm{~J} /\left(\mathrm{m}^{3} \mathrm{~K}\right)\right)[1]$. The $20 \%$ reduction on rolling, which is outside any experimental uncertainty, points towards a strong distortion of the phonon density which may be elucidated by an investigation of the change of the Debye temperature due to rolling. The quantities $\kappa$ and $\rho c$ are from the microscopic point of view partially decoupled as thermal conductivity $\kappa$ is governed by the heat transport via electrons in the frame of the Wiedemann-Franz law whereas the heat capacity is essentially based on the heat content of the phonon bath.

\section{Conclusion}

Frequency dependent photothermal radiometry has proven to be a suited tool to explore the impact of mechanical rolling on the thermal properties of $\mathrm{NiTi}$ foils. The thermal diffusivity and the thermal effusivity determined from the frequency dependent phase and amplitude of the thermal transmission signal suffer both from a considerable decrease with the rolling induced thickness change of the foil. The observed recovering of the thermal parameters after a heat treatment of the samples support the interpretation of defect induced changes of the thermal properties. The thermal conductivity deduced from the diffusivity and effusivity data display also a strong reduction with rolling which lies in the range of the values published for different NiTi alloy samples. The unexpected simultaneous decrease in the mass specific heat capacity with rolling points towards a considerable distortion of the phonon distribution which should be reflected in a decrease in the Debye temperature.

\section{Acknowledgments}

This work is performed in the frame of the Sonderforschungsbereich Shape Memory Technology (SFB 459). 


\section{References}

[1] J. van Humbeeck, Mater. Sci. Eng. A 273-275, 134 (1999).

[2] M. Kaack, I. Delgadillo-Holtfort, T. Yohannes, J. Pelzl, Mater. Sci. Eng. A 378, 119 (2004).

[3] J. Gibkes, A. AlHajDaoud, Ch. Zamponi, R. Wernhardt, B.K. Bein, J. Pelzl, in: Proc. Int. Conf. Shape Memory and Superelastic Technologies SMST-2004, Baden-Baden, Eds. M. Mertmann, H. Fischer, Trans Tech Publ., Zurich 2005. 